\title{
Corrigendum
}

\section{Corrigendum to "Observer-Based Adaptive Iterative Learning Control for a Class of Nonlinear Time Delay Systems with Input Saturation"}

\author{
Jian-ming Wei, Yun-an Hu, and Mei-mei Sun \\ Department of Control Engineering, Naval Aeronautical and Astronautical University, Yantai 264001, China \\ Correspondence should be addressed to Jian-ming Wei; wjm604@163.com \\ Received 15 October 2015; Accepted 3 November 2015
}

Copyright (C) 2015 Jian-ming Wei et al. This is an open access article distributed under the Creative Commons Attribution License, which permits unrestricted use, distribution, and reproduction in any medium, provided the original work is properly cited.

The purpose of this paper is to correct the errors in the paper titled "Observer-Based Adaptive Iterative Learning Control for a Class of Nonlinear Time Delay Systems with Input Saturation" [1]. In the adaptive learning law of $\widehat{W}_{k}(t)$ in (44) of [1], the variable $z_{k}$ was used. However in fact, $z_{k}$ is difficult to be obtained as the states are unmeasurable. The correction and some consequent modifications in the technical derivations are detailed as follows, while the main results are unchanged.

By Young's inequality, we can have

$$
\begin{aligned}
- & 2 z_{k}^{T} P B \widetilde{W}_{k}^{T} \phi\left(\widehat{x}_{k}\right) \\
= & -2 z_{k}^{T} C C^{T}\left(C C^{T}+\delta I_{n}\right)^{-1} P B \widetilde{W}_{k}^{T} \phi\left(\widehat{x}_{k}\right) \\
& -2 z_{k}^{T} \delta I_{n}\left(C C^{T}+\delta I_{n}\right)^{-1} P B \widetilde{W}_{k}^{T} \phi\left(\widehat{x}_{k}\right) \\
\leq & -2 z_{1, k} C^{T}\left(C C^{T}+\delta I_{n}\right)^{-1} P B \widetilde{W}_{k}^{T} \phi\left(\widehat{x}_{k}\right) \\
& +\frac{z_{k}^{T} P P^{T} z_{k}}{\left\|C C^{T}+\delta I_{n}\right\|^{2}}+\delta^{2} l \widetilde{W}_{k}^{T} \widetilde{W}_{k},
\end{aligned}
$$

where $\delta>0$ is a small positive constant.

Then (27) in [1] should be updated to

$$
\begin{aligned}
\dot{V}_{z_{k}} & \dot{V}_{U_{k}} \\
\leq & z_{k}^{T}\left(A^{T} P+P A+\frac{n+3}{\lambda} P P^{T}+\frac{P P^{T}}{\left\|C C^{T}+\delta I_{n}\right\|^{2}}\right) z_{k} \\
& -2 z_{1, k} C^{T}\left(C C^{T}+\delta I_{n}\right)^{-1} P B \widetilde{W}_{k}^{T} \phi\left(\widehat{x}_{k}\right)
\end{aligned}
$$

$$
\begin{aligned}
& +\frac{\lambda}{(1-\kappa)} \sum_{j=1}^{n} \rho_{j}^{2}\left(y_{k}\right)+\lambda D_{0}^{2}+4 \lambda l \varepsilon_{W}^{2}+\lambda \varepsilon_{0}^{2} \\
& +\delta^{2} l \widetilde{W}_{k}^{T} \widetilde{W}_{k} \\
\leq & -z_{k}^{T} Q z_{k}-2 z_{1, k} C^{T}\left(C C^{T}+\delta I_{n}\right)^{-1} P B \widetilde{W}_{k}^{T} \phi\left(\widehat{x}_{k}\right) \\
& +\frac{\lambda}{(1-\kappa)} \sum_{j=1}^{n} \rho_{j}^{2}\left(y_{k}\right)+\lambda D_{0}^{2}+4 \lambda \varepsilon_{W}^{2}+\lambda \varepsilon_{0}^{2} \\
& +\delta^{2} l \widetilde{W}_{k}^{T} \widetilde{W}_{k} \\
\leq & -\lambda_{\min }(Q)\left\|z_{k}\right\|^{2} \\
& -2 z_{1, k} C^{T}\left(C C^{T}+\delta I_{n}\right)^{-1} P B \widetilde{W}_{k}^{T} \phi\left(\widehat{x}_{k}\right) \\
& +\frac{\lambda}{(1-\kappa)} \sum_{j=1}^{n} \rho_{j}^{2}\left(y_{k}\right)+\lambda D_{0}^{2}+4 \lambda l \varepsilon_{W}^{2}+\lambda \varepsilon_{0}^{2} \\
& +\delta^{2} l \widetilde{W}_{k}^{T} \widetilde{W}_{k},
\end{aligned}
$$

where using updated inequality (12) of [1]

$$
A^{T} P+P A+\frac{n+3}{\lambda} P P^{T}+\frac{P P^{T}}{\left\|C C^{T}+\delta I_{n}\right\|^{2}}<-Q .
$$

Consequently, the derivative of $V_{k}$ should be revised as

$$
\begin{gathered}
\dot{V}_{k} \leq-\lambda_{\min }(Q)\left\|z_{k}\right\|^{2}-2 z_{1, k} C^{T}\left(C C^{T}+\delta I_{n}\right)^{-1} \\
\cdot P B \widetilde{W}_{k}^{T} \phi\left(\widehat{x}_{k}\right)+\delta^{2} l \widetilde{W}_{k}^{T} \widetilde{W}_{k}+s_{k}\left[\sum_{j=1}^{n-1} \lambda_{j} e_{j+1, k}\right.
\end{gathered}
$$




$$
\begin{aligned}
& +\left[\begin{array}{ll}
\Lambda^{T} & 1
\end{array}\right] K_{0} z_{1, k}+K e_{s k}-y_{d}^{(n)}+\widehat{W}_{k}^{T} \phi\left(\widehat{x}_{k}\right)+v_{k} \\
& \left.+\frac{b \tanh ^{2}\left(s_{k} / \eta(t)\right) \Xi\left(y_{k}\right)}{s_{k}}\right]+[1 \\
& \left.-b \tanh ^{2}\left(\frac{s_{k}}{\eta(t)}\right)\right] \Xi\left(y_{k}\right)-K s_{k}^{2} .
\end{aligned}
$$

In order to update $\widehat{W}_{k}(t)$ in the absence of $z_{k}$, we design the following differential-difference type learning law:

$$
\begin{aligned}
\left(1-\gamma_{1}\right) \dot{\widehat{W}}_{k}= & -\gamma_{1} \widehat{W}_{k}-\gamma_{1} \alpha \widehat{W}_{k}+\gamma_{1} \widehat{W}_{k-1} \\
& +2 q_{1} z_{1, k} C^{T}\left(C C^{T}+\delta I_{n}\right)^{-1} P B \phi\left(\widehat{x}_{k}\right), \\
\widehat{W}_{k}(0)= & \widehat{W}_{k-1}(T) \\
\widehat{W}_{0}(t)= & 0
\end{aligned}
$$$$
t \in[0, T] .
$$

The adaptive learning law for $\widehat{W}_{2, k}(t)$ is not changed, but some notations are updated, which is specified by

$$
\begin{aligned}
\left(1-\gamma_{2}\right) \dot{\widehat{W}}_{2, k}= & -\gamma_{2} \widehat{W}_{2, k}+\gamma_{2} \widehat{W}_{2, k-1} \\
& +q_{2} b \tanh ^{2}\left(\frac{s_{k}}{\eta(t)}\right) \phi_{2}\left(y_{k}\right), \\
\widehat{W}_{2, k}(0)= & \widehat{W}_{2, k-1}(T), \\
\widehat{W}_{2,0}(t)= & 0
\end{aligned}
$$$$
t \in[0, T],
$$

where $0<\gamma_{1}, \gamma_{2}<1$, and $\alpha_{1}>0$ are design parameters.

By substituting the controller back into (4), (46)-(47) of [1] should be replaced by

$$
\begin{aligned}
\dot{V}_{k} & \leq-\lambda_{\min }(Q)\left\|z_{k}\right\|^{2} \\
& -2 q_{1} z_{1, k} C^{T}\left(C C^{T}+\delta I_{n}\right)^{-1} P B \widetilde{W}_{k}^{T} \phi\left(\widehat{x}_{k}\right) \\
& +\delta^{2} l \widetilde{W}_{k}^{T} \widetilde{W}_{k}-b \widetilde{W}_{2, k}^{T} \phi_{2}\left(y_{k}\right) \tanh ^{2}\left(\frac{s_{k}}{\eta(t)}\right) \\
& +\left[1-b \tanh ^{2}\left(\frac{s_{k}}{\eta(t)}\right)\right] \Xi\left(y_{k}\right)-K s_{k}^{2}, \\
2 q_{1} & z_{1, k} C^{T}\left(C C^{T}+\delta I_{n}\right)^{-1} P B \widetilde{W}_{k}^{T} \phi\left(\widehat{x}_{k}\right) \\
& +b \widetilde{W}_{2, k}^{T} \phi_{2}\left(y_{k}\right) \tanh ^{2}\left(\frac{s_{k}}{\eta(t)}\right) \leq-\dot{V}_{k} \\
& -\lambda \min _{1}(Q)\left\|z_{k}\right\|^{2}+\left[1-b \tanh ^{2}\left(\frac{s_{k}}{\eta(t)}\right)\right] \Xi\left(y_{k}\right) \\
& -K s_{k}^{2}+\delta^{2} l \widetilde{W}_{k}^{T} \widetilde{W}_{k} .
\end{aligned}
$$

Accordingly, the Lyapunov-like CEF is updated to

$$
\begin{aligned}
E_{k}(t)= & \frac{\gamma_{1}}{2 q_{1}} \int_{0}^{t} \widetilde{W}_{k}^{T} \widetilde{W}_{k} \mathrm{~d} \sigma+\frac{\left(1-\gamma_{1}\right)}{2 q_{1}} \widetilde{W}_{k}^{T} \widetilde{W}_{k} \\
& +\frac{\gamma_{2}}{2 q_{2}} \int_{0}^{t} \widetilde{W}_{2, k}^{T} \widetilde{W}_{2, k} \mathrm{~d} \sigma+\frac{\left(1-\gamma_{2}\right)}{2 q_{2}} \widetilde{W}_{2, k}^{T} \widetilde{W}_{2, k} .
\end{aligned}
$$

The difference of $E_{k}(t)$ should be changed to

$$
\begin{aligned}
\Delta E_{k}(t)= & E_{k}(t)-E_{k-1}(t) \\
= & \frac{\gamma_{1}}{2 q_{1}} \int_{0}^{t}\left[\widetilde{W}_{k}^{T} \widetilde{W}_{k}-\widetilde{W}_{k-1}^{T} \widetilde{W}_{k-1}\right] \mathrm{d} \sigma \\
& +\frac{\left(1-\gamma_{1}\right)}{2 q_{1}}\left[\widetilde{W}_{k}^{T} \widetilde{W}_{k}-\widetilde{W}_{k-1}^{T} \widetilde{W}_{k-1}\right] \\
& +\frac{\gamma_{2}}{2 q_{2}} \int_{0}^{t}\left[\widetilde{W}_{2, k}^{T} \widetilde{W}_{2, k}-\widetilde{W}_{2, k-1}^{T} \widetilde{W}_{2, k-1}\right] \mathrm{d} \sigma \\
& +\frac{\left(1-\gamma_{2}\right)}{2 q_{2}}\left[\widetilde{W}_{2, k}^{T} \widetilde{W}_{2, k}-\widetilde{W}_{2, k-1}^{T} \widetilde{W}_{2, k-1}\right] .
\end{aligned}
$$

By using adaptive learning law (5) and inequality $2 \widetilde{W}_{k}^{T} \widehat{W}_{k} \geq \widetilde{W}_{k}^{T} \widetilde{W}_{k}-W^{* T} W^{*}$, we may have

$$
\begin{aligned}
& \frac{\gamma_{1}}{2 q_{1}} \int_{0}^{t}\left[\widetilde{W}_{k}^{T} \widetilde{W}_{k}-\widetilde{W}_{k-1}^{T} \widetilde{W}_{k-1}\right] \mathrm{d} \sigma \\
& +\frac{\left(1-\gamma_{1}\right)}{2 q_{1}}\left[\widetilde{W}_{k}^{T} \widetilde{W}_{k}-\widetilde{W}_{k-1}^{T} \widetilde{W}_{k-1}\right] \\
& =\frac{\gamma_{1}}{2 q_{1}} \int_{0}^{t}\left[\widetilde{W}_{k}^{T} \widetilde{W}_{k}-\widetilde{W}_{k-1}^{T} \widetilde{W}_{k-1}\right] \mathrm{d} \sigma \\
& +\frac{\left(1-\gamma_{1}\right)}{q_{1}} \int_{0}^{t} \widetilde{W}_{k}^{T} \dot{\widetilde{W}}_{k} \mathrm{~d} \sigma \\
& +\frac{\left(1-\gamma_{1}\right)}{2 q_{1}}\left[\widetilde{W}_{k}^{T}(0) \widetilde{W}_{k}(0)-\widetilde{W}_{k-1}^{T} \widetilde{W}_{k-1}\right] \\
& =\int_{0}^{t} 2 z_{1, k} C^{T}\left(C C^{T}+\delta I_{n}\right)^{-1} P B \widetilde{W}_{k}^{T} \phi\left(\widehat{x}_{k}\right) \mathrm{d} \sigma \\
& -\frac{\gamma_{1}}{q_{1}} \int_{0}^{t} \widetilde{W}_{k}^{T}\left(\alpha_{1} \widehat{W}_{k}+\widehat{W}_{k}-\widehat{W}_{k-1}\right) \mathrm{d} \sigma \\
& +\frac{\gamma_{1}}{2 q_{1}} \int_{0}^{t}\left[\widetilde{W}_{k}^{T} \widetilde{W}_{k}-\widetilde{W}_{k-1}^{T} \widetilde{W}_{k-1}\right] \mathrm{d} \sigma \\
& +\frac{\left(1-\gamma_{1}\right)}{2 q_{1}}\left[\widetilde{W}_{k}^{T}(0) \widetilde{W}_{k}(0)-\widetilde{W}_{k-1}^{T} \widetilde{W}_{k-1}\right] \\
& =\int_{0}^{t} 2 z_{1, k} C^{T}\left(C C^{T}+\delta I_{n}\right)^{-1} P B \widetilde{W}_{k}^{T} \phi\left(\widehat{x}_{k}\right) \mathrm{d} \sigma \\
& -\frac{\alpha_{1} \gamma_{1}}{q_{1}} \int_{0}^{t} \widetilde{W}_{k}^{T} \widehat{W}_{k} \mathrm{~d} \sigma-\frac{\gamma_{1}}{q_{1}} \int_{0}^{t} \widetilde{W}_{k}^{T}\left(\widetilde{W}_{k}-\widetilde{W}_{k-1}\right) \mathrm{d} \sigma \\
& +\frac{\gamma_{1}}{2 q_{1}} \int_{0}^{t}\left[\widetilde{W}_{k}^{T} \widetilde{W}_{k}-\widetilde{W}_{k-1}^{T} \widetilde{W}_{k-1}\right] \mathrm{d} \sigma
\end{aligned}
$$




$$
\begin{aligned}
& +\frac{\left(1-\gamma_{1}\right)}{2 q_{1}}\left[\widetilde{W}_{k}^{T}(0) \widetilde{W}_{k}(0)-\widetilde{W}_{k-1}^{T} \widetilde{W}_{k-1}\right] \\
& =\int_{0}^{t} 2 z_{1, k} C^{T}\left(C C^{T}+\delta I_{n}\right)^{-1} P B \widetilde{W}_{k}^{T} \phi\left(\widehat{x}_{k}\right) \mathrm{d} \sigma \\
& -\frac{\alpha_{1} \gamma_{1}}{q_{1}} \int_{0}^{t} \widetilde{W}_{k}^{T} \widehat{W}_{k} \mathrm{~d} \sigma \\
& -\frac{\gamma_{1}}{2 q_{1}} \int_{0}^{t}\left(\widetilde{W}_{k}-\widetilde{W}_{k-1}\right)^{T}\left(\widetilde{W}_{k}-\widetilde{W}_{k-1}\right) \mathrm{d} \sigma \\
& +\frac{\left(1-\gamma_{1}\right)}{2 q_{1}}\left[\widetilde{W}_{k}^{T}(0) \widetilde{W}_{k}(0)-\widetilde{W}_{k-1}^{T} \widetilde{W}_{k-1}\right] \\
& \leq \int_{0}^{t} 2 z_{1, k} C^{T}\left(C C^{T}+\delta I_{n}\right)^{-1} P B \widetilde{W}_{k}^{T} \phi\left(\widehat{x}_{k}\right) \mathrm{d} \sigma \\
& -\frac{\alpha_{1} \gamma_{1}}{2 q_{1}} \int_{0}^{t} \widetilde{W}_{k}^{T} \widetilde{W}_{k} \mathrm{~d} \sigma+\frac{\alpha_{1} \gamma_{1}}{2 q_{1}} \int_{0}^{t}\left\|W^{*}(\sigma)\right\| \mathrm{d} \sigma \\
& +\frac{\left(1-\gamma_{1}\right)}{2 q_{1}}\left[\widetilde{W}_{k}^{T}(0) \widetilde{W}_{k}(0)-\widetilde{W}_{k-1}^{T} \widetilde{W}_{k-1}\right] .
\end{aligned}
$$

Recalling (8), inequality (B.8) of [1] is changed to $\Delta E_{k}(t)$

$$
\begin{aligned}
\leq & \int_{0}^{t} 2 z_{k}^{T} P B \widetilde{W}_{k}^{T} \phi\left(\widehat{x}_{k}\right) \mathrm{d} \sigma \\
& +\int_{0}^{t} b \tanh ^{2}\left(\frac{s_{k}}{\eta}\right) \widetilde{W}_{2, k}^{T} \phi_{2}\left(y_{k}\right) \mathrm{d} \sigma \\
& +\frac{\alpha_{1} \gamma_{1}}{2 q_{1}} \int_{0}^{t}\left\|W^{*}(\sigma)\right\| \mathrm{d} \sigma-\frac{\alpha_{1} \gamma_{1}}{2 q_{1}} \int_{0}^{t} \widetilde{W}_{k}^{T} \widetilde{W}_{k} \mathrm{~d} \sigma \\
& +\frac{\left(1-\gamma_{1}\right)}{2 q_{1}}\left[\widetilde{W}_{k}^{T}(0) \widetilde{W}_{k}(0)-\widetilde{W}_{k-1}^{T} \widetilde{W}_{k-1}\right] \\
& +\frac{\left(1-\gamma_{2}\right)}{2 q_{2}}\left[\widetilde{W}_{2, k}^{T}(0) \widetilde{W}_{2, k}(0)-\widetilde{W}_{2, k-1}^{T} \widetilde{W}_{2, k-1}\right] \\
\leq & -V_{k}(t)+V_{k}(0)-K \int_{0}^{t} s_{k}^{2} \mathrm{~d} \sigma \\
& -\lambda_{\min }(Q) \int_{0}^{t}\left\|z_{k}\right\|^{2} \mathrm{~d} \sigma \\
& -\int_{0}^{t}\left(\frac{\alpha_{1} \gamma_{1}}{2 q_{1}}-l \delta^{2}\right) \widetilde{W}_{k}^{T} \widetilde{W}_{k} \mathrm{~d} \sigma \\
& +\frac{\alpha_{1} \gamma_{1}}{2 q_{1}} \int_{0}^{t}\left\|W^{*}(\sigma)\right\| \mathrm{d} \sigma \\
& +\frac{\left(1-\gamma_{1}\right)}{2 q_{1}}\left[\widetilde{W}_{k}^{T}(0) \widetilde{W}_{k}(0)-\widetilde{W}_{k-1}^{T} \widetilde{W}_{k-1}\right] \\
& +\frac{\left(1-\gamma_{2}\right)}{2 q_{2}}\left[\widetilde{W}_{2, k}^{T}(0) \widetilde{W}_{2, k}(0)-\widetilde{W}_{2, k-1}^{T} \widetilde{W}_{2, k-1}\right] .
\end{aligned}
$$

Choose suitable design parameters such that $\alpha_{1} \gamma_{1} / 2 q_{1}-$ $l \delta^{2}>0$. Then it follows from (12) that

$\Delta E_{k}(t)$

$$
\begin{aligned}
\leq & -V_{k}(t)+V_{k}(0)-K \int_{0}^{t} s_{k}^{2} \mathrm{~d} \sigma \\
& -\lambda_{\min }(Q) \int_{0}^{t}\left\|z_{k}\right\|^{2} \mathrm{~d} \sigma+\frac{\alpha_{1} \gamma_{1}}{2 q_{1}} \int_{0}^{t}\left\|W^{*}(\sigma)\right\| \mathrm{d} \sigma \\
& +\frac{\left(1-\gamma_{1}\right)}{2 q_{1}}\left[\widetilde{W}_{k}^{T}(0) \widetilde{W}_{k}(0)-\widetilde{W}_{k-1}^{T} \widetilde{W}_{k-1}\right] \\
& +\frac{\left(1-\gamma_{2}\right)}{2 q_{2}}\left[\widetilde{W}_{2, k}^{T}(0) \widetilde{W}_{2, k}(0)-\widetilde{W}_{2, k-1}^{T} \widetilde{W}_{2, k-1}\right] .
\end{aligned}
$$

Consequently, (B.9) of [1] is updated to

$$
\begin{aligned}
\Delta E_{k}(T) \leq & -V_{k}(T)-K \int_{0}^{T} s_{k}^{2} \mathrm{~d} \sigma \\
& -\lambda_{\min }(Q) \int_{0}^{T}\left\|z_{k}\right\|^{2} \mathrm{~d} \sigma \\
& +\frac{\alpha_{1} \gamma_{1}}{2 q_{1}} \int_{0}^{t}\left\|W^{*}(\sigma)\right\| \mathrm{d} \sigma \\
\leq & -K \int_{0}^{T} s_{k}^{2} \mathrm{~d} \sigma-\lambda_{\min }(Q) \int_{0}^{T}\left\|z_{k}\right\|^{2} \mathrm{~d} \sigma \\
& +\frac{\alpha_{1} \gamma_{1}}{2 q_{1}} \int_{0}^{t}\left\|W^{*}(\sigma)\right\| \mathrm{d} \sigma .
\end{aligned}
$$

By using adaptive learning law $\left(1-\gamma_{1}\right) \dot{\widehat{W}}_{1}=-\gamma_{1} \widehat{W}_{1}-$ $\gamma_{1} \alpha_{1} \widehat{W}_{1}+2 q_{1} z_{1,1} C^{T}\left(C C^{T}+\delta I_{n}\right)^{-1} P B \phi\left(\widehat{x}_{1}\right)$, it is clear that

$$
\begin{aligned}
& \frac{\gamma_{1}}{2 q_{1}} \widetilde{W}_{1}^{T} \widetilde{W}_{1}+\frac{\left(1-\gamma_{1}\right)}{q_{1}} \widetilde{W}_{1}^{T} \dot{W}_{1} \\
&=\frac{\gamma_{1}}{2 q_{1}} \widetilde{W}_{1}^{T} \widetilde{W}_{1}-\frac{\gamma_{1}}{q_{1}} \widetilde{W}_{1}^{T} \widehat{W}_{1}-\frac{\alpha_{1} \gamma_{1}}{q_{1}} \widetilde{W}_{1}^{T} \widehat{W}_{1} \\
& \quad+2 z_{1, k} C^{T}\left(C C^{T}+\delta I_{n}\right)^{-1} P B \widetilde{W}_{k}^{T} \phi\left(\widehat{x}_{k}\right) \\
&=\frac{\gamma_{1}}{2 q_{1}}\left[\widetilde{W}_{1}^{T} \widetilde{W}_{1}-2 \widetilde{W}_{1}^{T} \widehat{W}_{1}+\widehat{W}_{1}^{T} \widehat{W}_{1}\right]-\frac{\gamma_{1}}{2 q_{1}} \widehat{W}_{1}^{T} \widehat{W}_{1} \\
&-\frac{\alpha_{1} \gamma_{1}}{q_{1}} \widetilde{W}_{1}^{T} \widehat{W}_{1} \\
&+2 z_{1,1} C^{T}\left(C C^{T}+\delta I_{n}\right)^{-1} P B \widetilde{W}_{1}^{T} \phi\left(\widehat{x}_{1}\right) \\
& \leq \frac{\gamma_{1}}{2 q_{1}}\left[\widehat{W}_{1}-\widetilde{W}_{1}\right]^{T}\left[\widehat{W}_{1}-\widetilde{W}_{1}\right]-\frac{\alpha_{1} \gamma_{1}}{2 q_{1}} \widetilde{W}_{1}^{T} \widetilde{W}_{1} \\
&+\frac{\alpha_{1} \gamma_{1}}{2 q_{1}} W^{* T} W^{*} \\
&+2 z_{1,1} C^{T}\left(C C^{T}+\delta I_{n}\right)^{-1} P B \widetilde{W}_{1}^{T} \phi\left(\widehat{x}_{1}\right)
\end{aligned}
$$




$$
\begin{aligned}
\leq & \left(1+\alpha_{1}\right) \frac{\gamma_{1}}{2 q_{1}} W^{* T} W^{*} \\
& +2 z_{1,1} C^{T}\left(C C^{T}+\delta I_{n}\right)^{-1} P B \widetilde{W}_{1}^{T} \phi\left(\widehat{x}_{1}\right) \\
& -\frac{\alpha_{1} \gamma_{1}}{2 q_{1}} \widetilde{W}_{1}^{T} \widetilde{W}_{1} .
\end{aligned}
$$

Thus $\dot{E}_{1}(t)$ is changed to

$$
\begin{aligned}
\dot{E}_{1}(t) \leq & \left(1+\alpha_{1}\right) \frac{\gamma_{1}}{2 q_{1}} W^{* T} W^{*}+\frac{\gamma_{2}}{2 q_{2}} W_{2}^{* T} W_{2}^{*} \\
& +2 z_{1, k} C^{T}\left(C C^{T}+\delta I_{n}\right)^{-1} P B \widetilde{W}_{1}^{T} \phi\left(\widehat{x}_{1}\right) \\
& +b \tanh ^{2}\left(\frac{s_{1}}{\eta(t)}\right) \widetilde{W}_{2,1}^{T} \phi_{2}\left(y_{1}\right)-\frac{\alpha_{1} \gamma_{1}}{2 q_{1}} \widetilde{W}_{1}^{T} \widetilde{W}_{1} \\
\leq & -\dot{V}_{1}-\lambda_{\min }(Q)\left\|z_{1}\right\|^{2}-K s_{1}^{2} \\
& +\left(1+\alpha_{1}\right) \frac{\gamma_{1}}{2 q_{1}} W^{* T} W^{*}+\frac{\gamma_{2}}{2 q_{2}} W_{2}^{* T} W_{2}^{*} \\
& -\left(\frac{\alpha_{1} \gamma_{1}}{2 q_{1}}-l \delta^{2}\right) \widetilde{W}_{1}^{T} \widetilde{W}_{1} \\
\leq & -\dot{V}_{1}-\lambda_{\min }(Q)\left\|z_{1}\right\|^{2}-K s_{1}^{2} \\
& +\left(1+\alpha_{1}\right) \frac{\gamma_{1}}{2 q_{1}} W^{* T} W^{*}+\frac{\gamma_{2}}{2 q_{2}} W_{2}^{* T} W_{2}^{*} .
\end{aligned}
$$

Denote $c_{\max }=\max _{t \in[0, T]}\left\{\left(1+\alpha_{1}\right)\left(\gamma_{1} / 2 q_{1}\right) W^{* T}(t) W^{*}(t)+\right.$ $\left.\left(\gamma / 2 q_{2}\right) W_{2}^{* T} W_{2}^{*}\right\}$. The integral of $\dot{E}_{1}(t)$ over $[0, t]$ is updated as follows:

$$
\begin{aligned}
E_{1}(t)-E_{1}(0) \leq & -V_{1}(t)+V_{1}(0) \\
& -\lambda_{\min }(Q) \int_{0}^{t}\left\|z_{1}\right\|^{2} \mathrm{~d} \sigma \\
& -\int_{0}^{t} K s_{1}^{2}(\sigma) \mathrm{d} \sigma+t \cdot c_{\max } .
\end{aligned}
$$

According to the new definition of $\operatorname{CEF}(9), E_{1}(0)$ should be computed as follows:

$$
\begin{aligned}
E_{1}(0)= & \frac{\left(1-\gamma_{1}\right)}{2 q_{1}} \widetilde{W}_{1}^{T}(0) \widetilde{W}_{1}(0) \\
& +\frac{\left(1-\gamma_{2}\right)}{2 q_{2}} \widetilde{W}_{2,1}^{T}(0) \widetilde{W}_{2,1}(0) \\
= & \frac{\left(1-\gamma_{1}\right)}{2 q_{1}}\left\|W^{*}\right\|^{2}+\frac{\left(1-\gamma_{2}\right)}{2 q_{2}}\left\|W_{2}^{*}\right\|^{2} .
\end{aligned}
$$

Hence $E_{1}(T)$ is bounded by

$$
\begin{aligned}
E_{1}(T) & \leq T \cdot c_{\max }+\frac{\left(1-\gamma_{1}\right)}{2 q_{1}}\left\|W^{*}\right\|^{2}+\frac{(1-\gamma)}{2 q_{2}}\left\|W_{2}^{*}\right\|^{2} \\
& <\infty .
\end{aligned}
$$

We choose $\alpha_{1}=\Delta_{k}$, with $\left\{\Delta_{k}\right\}$ being a convergent series, which is defined by $\Delta_{k}=q / k^{l}$, where $l$ and $q$ are design parameters, $q(\in R)>0, l\left(\in Z_{+}\right) \geq 2 . \Delta_{k}$ has the following property.

Property 1 (see S. Zhu, M. X. Sun, and X. X. He, "Iterative learning control of strict-feedback nonlinear time-varying systems," Acta Automatica Sinica, vol. 36, no. 3, pp. 454-458, 2010). $\lim _{k \rightarrow \infty} \sum_{j=1}^{k} \Delta_{j} \leq 2 q$.

Using (14), it is followed by

$$
\begin{aligned}
E_{k}(T)= & E_{1}(T)+\sum_{j=2}^{k} \Delta E_{j}(T) \\
\leq & E_{1}(T)-K \sum_{j=2}^{k} \int_{0}^{T} s_{j}^{2} \mathrm{~d} \sigma \\
& -\lambda_{\min }(Q) \sum_{j=2}^{k} \int_{0}^{T}\left\|z_{j}\right\|^{2} \mathrm{~d} \sigma \\
& +\frac{\gamma_{1}}{2 q_{1}} \int_{0}^{T}\left\|W^{*}(\sigma)\right\| \mathrm{d} \sigma \sum_{j=2}^{k} \Delta_{k} .
\end{aligned}
$$

According to Property 1, we know $\sum_{j=1}^{k} \Delta_{k} \leq$ $\lim _{k \rightarrow \infty} \sum_{j=1}^{k} \Delta_{k} \leq 2 q$, which implies the boundedness of $E_{k}(T)$.

In the derivation of finiteness of $E_{k}(t)$, the changes are specified as follows.

Separate $E_{k}(t)$ into two parts:

$$
\begin{aligned}
& E_{k}^{1}(t)=\frac{\gamma_{1}}{2 q_{1}} \int_{0}^{t} \widetilde{W}_{k}^{T} \widetilde{W}_{k} \mathrm{~d} \sigma+\frac{\gamma_{2}}{2 q_{2}} \int_{0}^{t} \widetilde{W}_{2, k}^{T} \widetilde{W}_{2, k} \mathrm{~d} \sigma \\
& E_{k}^{2}(t)=\frac{\left(1-\gamma_{1}\right)}{2 q_{1}} \widetilde{W}_{k}^{T} \widetilde{W}_{k}+\frac{\left(1-\gamma_{2}\right)}{2 q_{2}} \widetilde{W}_{2, k}^{T} \widetilde{W}_{2, k} .
\end{aligned}
$$

The boundedness of $E_{k}^{1}(T)$ and $E_{k}^{2}(T)$ is guaranteed $\forall k \epsilon$ $N$. Thus, there exist two positive constants $M_{1}$ and $M_{2}$ satisfying

$$
\begin{aligned}
& E_{k}^{1}(t) \leq E_{k}^{1}(T) \leq M_{1}<\infty, \\
& E_{k}^{2}(T) \leq M_{2} .
\end{aligned}
$$

Consequently,

$$
E_{k}(t)=E_{k}^{1}(t)+E_{k}^{2}(t) \leq M_{1}+E_{k}^{2}(t) .
$$

On the other hand, it follows from (13) that

$$
\begin{aligned}
& \Delta E_{k+1}(t) \\
& <\frac{\Delta_{k} \gamma_{1}}{2 q_{1}} \int_{0}^{t}\left\|W^{*}(\sigma)\right\| \mathrm{d} \sigma \\
& \quad+\frac{\left(1-\gamma_{1}\right)}{2 q_{1}}\left[\widetilde{W}_{k}^{T}(0) \widetilde{W}_{k}(0)-\widetilde{W}_{k-1}^{T} \widetilde{W}_{k-1}\right]
\end{aligned}
$$




$$
\begin{aligned}
& +\frac{\left(1-\gamma_{2}\right)}{2 q_{2}}\left[\widetilde{W}_{2, k}^{T}(0) \widetilde{W}_{2, k}(0)-\widetilde{W}_{2, k-1}^{T} \widetilde{W}_{2, k-1}\right] \\
\leq & \frac{\Delta_{k} \gamma_{1}}{2 q_{1}} \int_{0}^{t}\left\|W^{*}(\sigma)\right\| \mathrm{d} \sigma+M_{2}-E_{k}^{2}(t) .
\end{aligned}
$$

Combining (23) and (24) results in

$$
\begin{aligned}
E_{k+1}(t) & =E_{k}(t)+\Delta E_{k+1}(t) \\
& \leq M_{1}+M_{2}+\frac{\Delta_{k} \gamma_{1}}{2 q_{1}} \int_{0}^{t}\left\|W^{*}(\sigma)\right\| \mathrm{d} \sigma .
\end{aligned}
$$

Since we have proved the boundedness of $E_{1}(t)$, the finiteness of $E_{k}(t)$ can be deduced by induction method.

Finally, we give the necessary revisions for the proof of convergence of tracking errors.

We can obtain from (20) that

$$
\begin{aligned}
& \sum_{j=2}^{k} \int_{0}^{T} s_{j}^{2} \mathrm{~d} \sigma \\
& \leq \frac{\left(E_{1}(T)-E_{k}(T)+\left(\gamma_{1} / 2 q_{1}\right) \int_{0}^{T}\left\|W^{*}(\sigma)\right\| \mathrm{d} \sigma \sum_{j=2}^{k} \Delta_{k}\right)}{K}, \\
& \sum_{j=2}^{k} \int_{0}^{T}\left\|z_{j}\right\|^{2} \mathrm{~d} \sigma \\
& \leq \frac{\left(E_{1}(T)-E_{k}(T)+\left(\gamma_{1} / 2 q_{1}\right) \int_{0}^{T}\left\|W^{*}(\sigma)\right\| \mathrm{d} \sigma \sum_{j=2}^{k} \Delta_{k}\right)}{\lambda_{\min }(Q)} .
\end{aligned}
$$

Considering Property 1 and taking the limitation of the above two inequalities yield

$$
\begin{aligned}
\lim _{k \rightarrow \infty} \sum_{j=2}^{k} \int_{0}^{T} s_{j}^{2}(\sigma) \mathrm{d} \sigma \\
\leq \frac{\left(E_{1}(T)+\left(\gamma_{1} q / q_{1}\right) \int_{0}^{T}\left\|W^{*}(\sigma)\right\| \mathrm{d} \sigma\right)}{K}, \\
\lim _{k \rightarrow \infty} \sum_{j=2}^{k} \int_{0}^{T}\left\|z_{j}\right\|^{2} \mathrm{~d} \sigma \\
\leq \frac{\left(E_{1}(T)+\left(\gamma_{1} q / q_{1}\right) \int_{0}^{T}\left\|W^{*}(\sigma)\right\| \mathrm{d} \sigma\right)}{\lambda_{\min }(Q)} .
\end{aligned}
$$

Similarly, according to the convergence of the sum of series, we can obtain the convergence of errors. The other parts are not changed.

\section{References}

[1] J.-m. Wei, Y.-a. Hu, and M.-m. Sun, "Observer-based adaptive iterative learning control for a class of nonlinear time delay systems with input saturation," Mathematical Problems in Engineering, vol. 2015, Article ID 645161, 19 pages, 2015. 


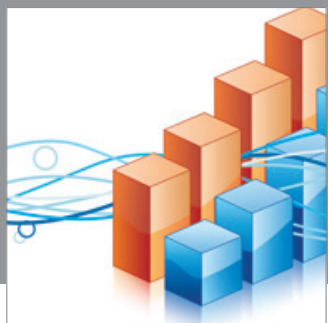

Advances in

Operations Research

mansans

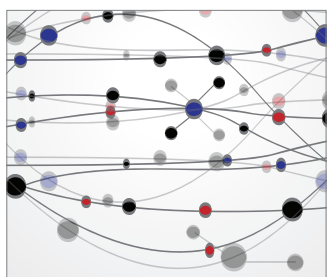

The Scientific World Journal
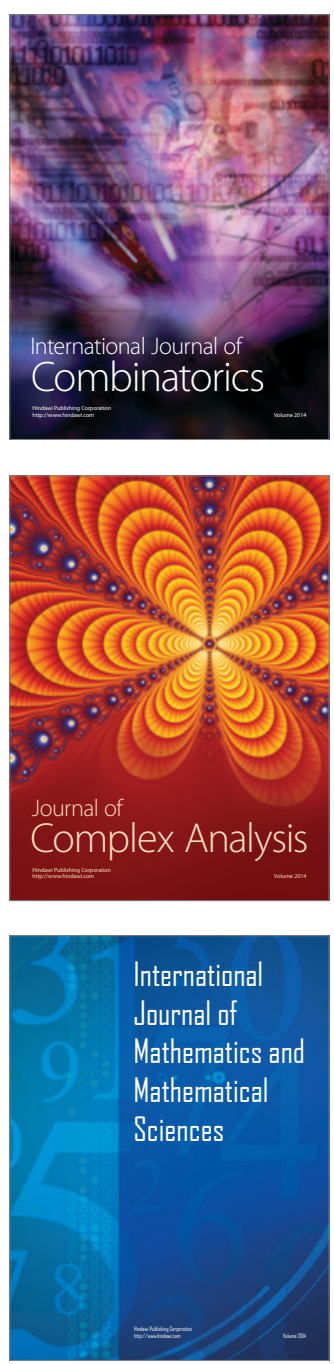
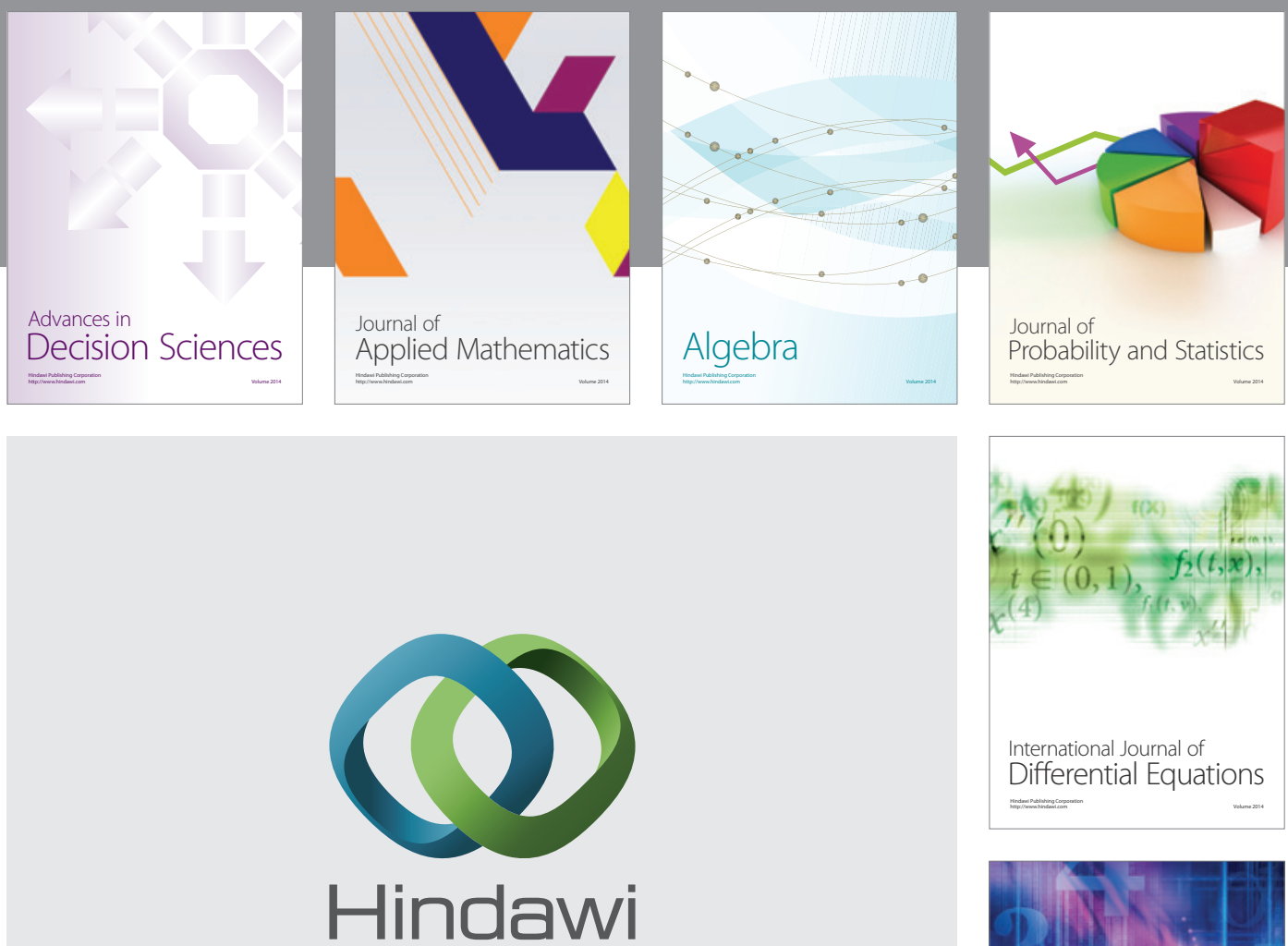

Submit your manuscripts at http://www.hindawi.com
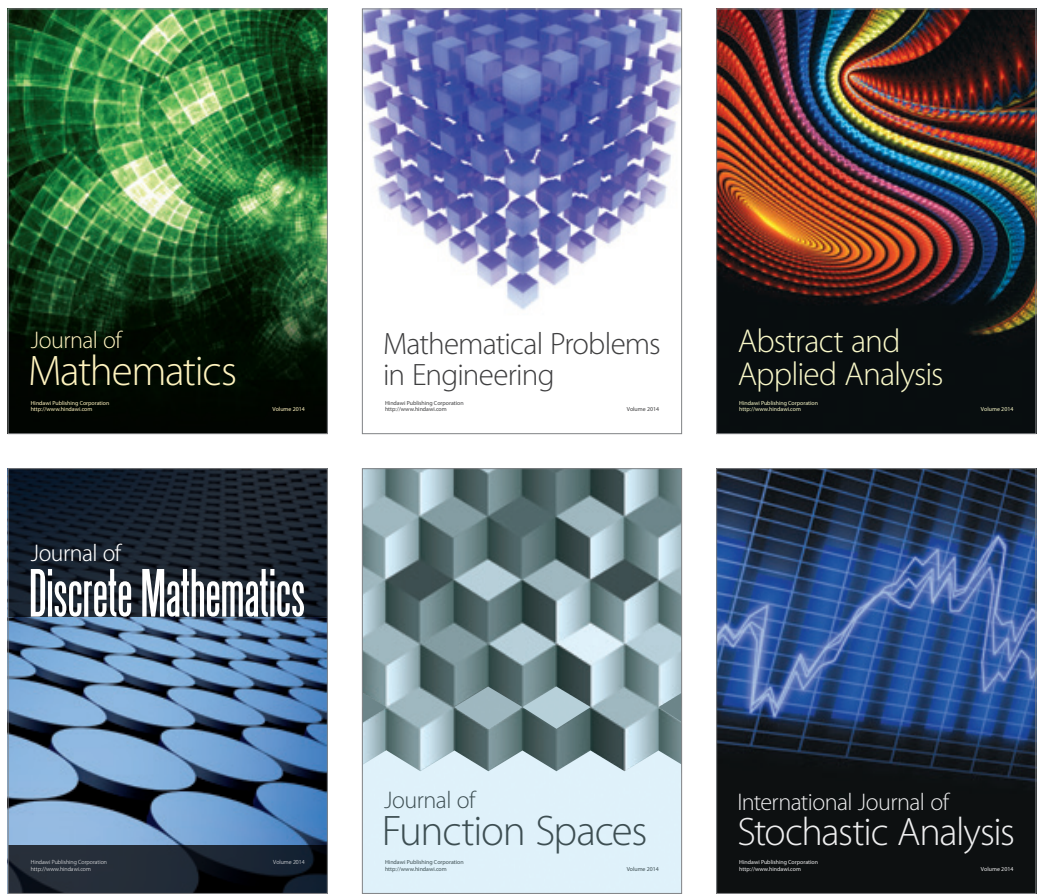

Journal of

Function Spaces

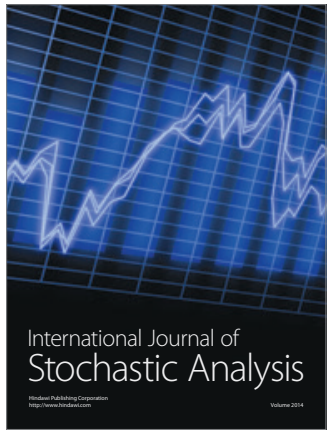

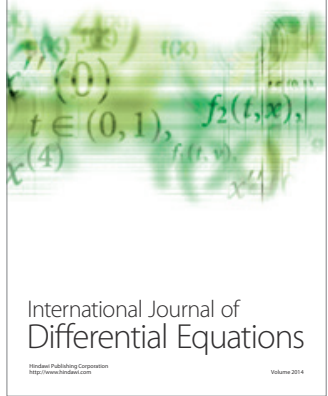
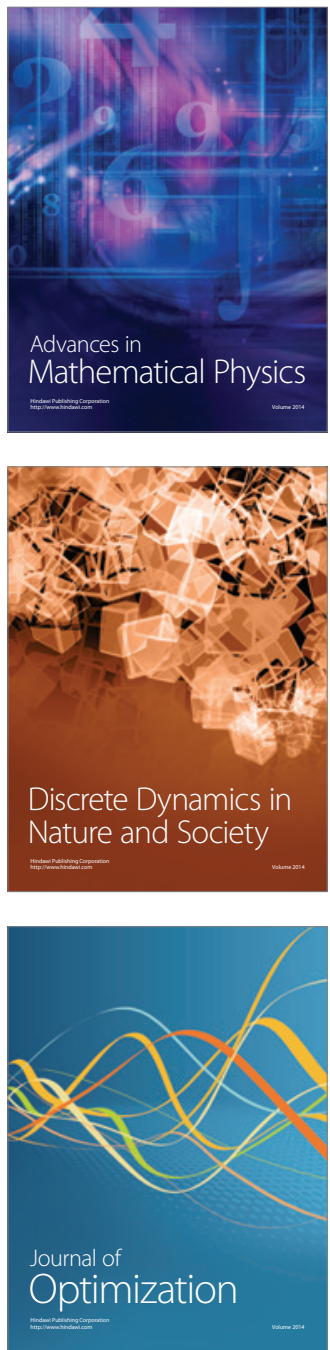